\title{
Antimicrobial resistance: The never ending story
}

\author{
LINDSEY E NICOLLE, MD
}

T HE THERAPEUTIC TRIUMPHS OF ANTIMICROBIALS IN the past decades have been echoed by the successes of microbial resistance to chemotherapy (1). Penicillin G therapy presaged hospital outbreaks of penicillin-resistant Staphylococcus aureus significant enough to lead to the foundation of hospital infection programs. On the heels of the introduction of ampicillin was ampicillin-resistant Haemophilus influenzae; optimism in the extended spectrum cephalosporins such as cefotaxime or ceftriaxone was immediately cooled by chromosomally inducible beta-lactamases produced by Enterobacter cloaceae, among others (2); and widespread ciprofloxacin-resistant, methicillin-resistant $S$ aureus appeared immediately with ciprofloxacin use for this organism. While certain organisms - the group A streptococcus and Neisseria meningitidis, for example - have remained susceptible to such 'early antibiotics' as penicillin $\mathrm{G}$, there can be no complacency. These bacteria have become resistant to other antimicrobials, such as erythromycin or sulphonamides, and we are simply waiting for the other shoe to drop. The increasing use of antiviral and antifungal chemotherapy has broadened the field. Acyclovir-resistant herpes simplex and azidothymidine-resistant human immunodeficiency virus are therapeutic problems; and prophylactic fluconazole therapy has seen the emergence of resistant Candida kruzeii.

Antimicrobial therapy alters bacterial ecosystems in at least two ways. As the examples above demonstrate, susceptible organisms may become resistant, with most strains being replaced by resistant clones. Today, penicillin-susceptible $S$ aureus is a microbiological dinosaur, and a source

Correspondence and reprints: Dr LE Nicolle, MS-675D. Health Sciences Centre, 820 Sherbrook Street, Winnipeg. Manitoba R3A 1 R9 of amusement when identified. Alternately, an ecological niche occupied by susceptible organisms is replaced by intrinsically resistant organisms. Health care interventions and widespread use of cephalosporins and related antibiotics have propelled coagulase negative staphylococci and Enterococcus faecalis to the forefront of hospitalacquired pathogens. Now we grapple with the problems of vancomycin-resistant Gram-positive organisms (2).

The contributions of anti-infective chemotherapy to health care should not be underestimated. The extraordinary adaptability, diversity and molecular flexibility of microorganisms, however, ensures that resistance will emerge to limit the usefulness of any antimicrobial. We are running hard to stay in the same place, but the treadmill is of our own creation. Societal and economic imperatives dictate that the pharmaceutical industry focus on new drug development, not old drug preservation. A system perpetually requiring 'new and improved' antimicrobials, largely because of emergence of resistance in microorganisms, serves both industry and academia well.

Is there an alternate approach? One repeated recommendation, whose proponents point to numerous studies documenting that less than 50\% of antimicrobials are used appropriately, is to restrict antimicrobial use (3). Encourage formulary restriction and control in acute care institutions. No antimicrobials in animal feed. Stop the selling of antimicrobials 'over the counter' in developing countries. Ecological modification through antimicrobial limitation has been documented to be effective, especially in acute care institutions (4). More widespread restriction on a societal basis would, however, be problematic. The restrictive approach is inconsistent with our western, 'free market' philosophy, which emphasizes individual rights and freedoms. Even if all 
inappropriate use of antimicrobials ceased, the expansion of certain high use patient populations, eg, bone marrow transplant patients, will require increasingly intensive antimicrobial use. In these populations, resistance will continue to emerge even with appropriate antimicrobial therapy. Thus, potential gains from the restrictive approach are not guaranteed. This does not mean that efforts to promote optimal antimicrobial use should not be pursued. It is likely naïve, however, to expect even completely optimal use to do more than slow the emergence of resistance. The arguments for optimal use of antimicrobials are primarily those of clinical care, adverse effects and cost, rather than resistance.

An alternate approach is to beat the bugs at their own game: to use molecular strategies to reverse or prevent microbial resistance. One can envisage the creation of transposons which provide some benefit to the organism, ensuring their acquisition and dissemination, but which also interfere with resistance mechanisms. While this is an attractive approach, it remains theoretical. One assumes that microorganisms would develop the means to circumvent these strategies, adding another level to the treadmill. A conceptually similar approach, the use of saprophytic organisms to fill the ecological vacuums created by antimicrobial use, has a long history, but there has been limited success and no current reason for optimism regarding the ultimate value of this approach.

The most effective means of preventing or delaying development of antimicrobial resistance must be the prevention of infection. Antimicrobials themselves may be used to prevent infection, but this use may lead to emergence of resistant organisms - this approach requires a cost-benefit analysis and further study. But the stage is larger.
Programs such as continued development and wider use of vaccine, and ensuring optimal hospital infection control are crucial to preserving the efficacy of antimicrobial agents. There remains room for advances in both of these areas. Provision of adequate nutrition, sanitation and housing should all be considered strategies to prevent the emergence of resistant organisms. Antimicrobial resistance is one issue in the distribution and appropriate use of resources in our own and other societies.

This editorial is not an attempt to reduce a complex issue to a simplistic concept. Despite universal problems with antimicrobial resistance, excellent antimicrobial therapy is still effective in managing most infections. Cure is determined more by host variables rather than the infecting organism. For individuals working in the field of infectious disease, the challenges of antimicrobial resistance provide daily intellectual and therapeutic stimulation. Issues of antimicrobial resistance are, however, one facet of the larger, ultimate goal of infectious diseases - prevention.

\section{REFERENCES}

1. Mayer KH, Opal SM, Medeiros AA. Mechanisms of antibiotic resistance. In: Mandell GL, Douglas RG Jr, Bennett JE, eds. Principles and Practice of Infectious Diseases, 3rd edn. New York: Churchill Livingstone Inc, 1990:218-28.

2. Jacoby GA, Archer GL. New mechanisms of bacterial resistance to antimicrobial agents. N Engl J Med 1991;324:601-12.

3. Marr JJ, Moffet HL, Kunin CM. Guidelines for improving the use of antimicrobial agents in hospitals. A statement by the Infectious Diseases Society of America. J Infect Dis 1988; 157:859-76.

4. McGowan JE Jr. Antimicrobial resistance in hospital organisms and its relation to antibiotic use. Rev Infect Dis 1983;5:1033-48. 


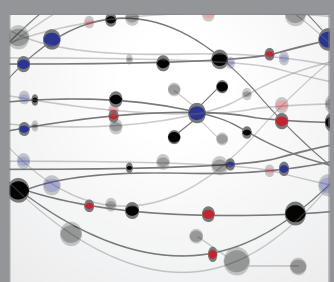

The Scientific World Journal
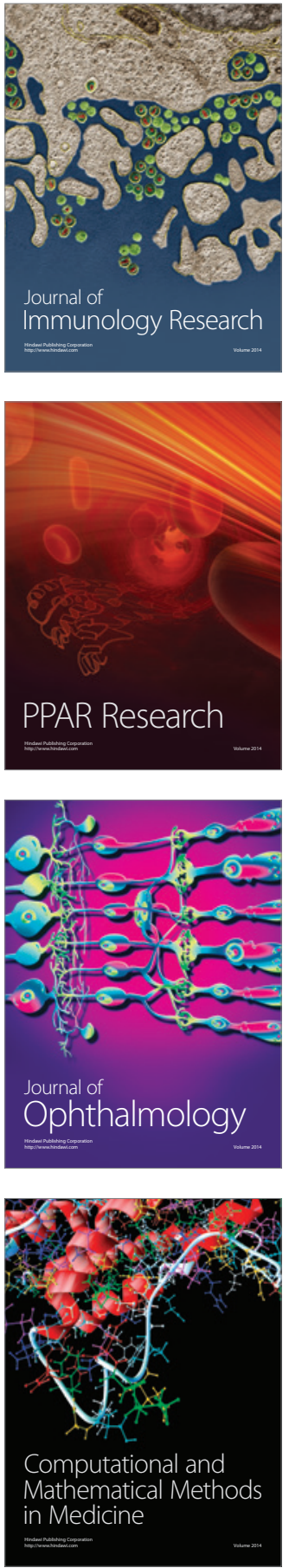

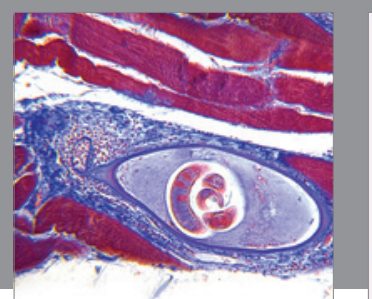

Gastroenterology Research and Practice

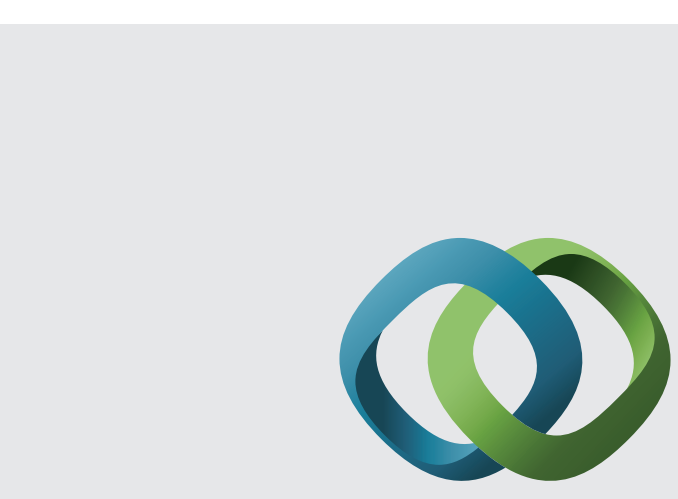

\section{Hindawi}

Submit your manuscripts at

http://www.hindawi.com
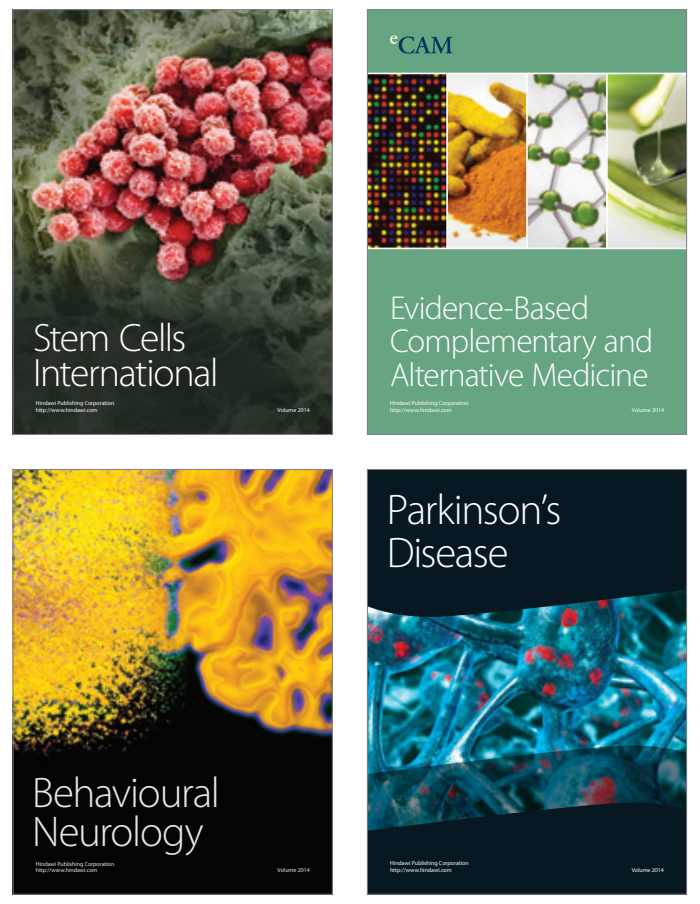
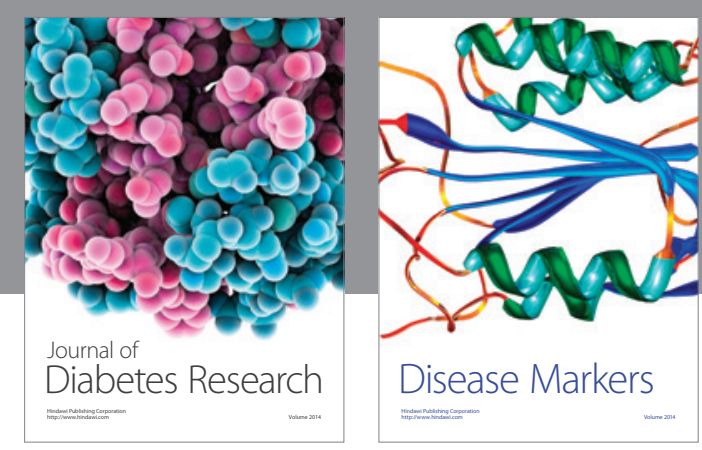

Disease Markers
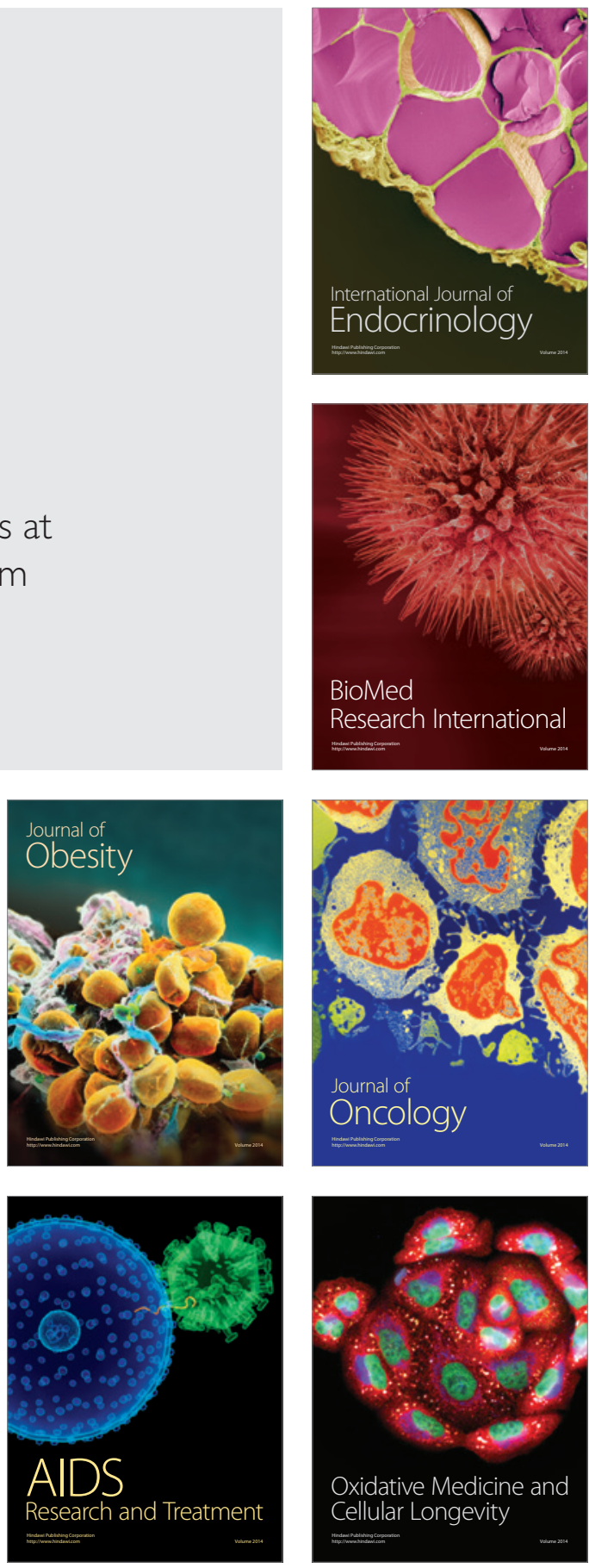